\title{
Application of a sensor array based on capillary-attached conductive gas sensors for odor identification
}

\begin{abstract}
An electronic nose based on an array of capillary-attached conductive gas sensors was fabricated. The identification ability of the developed structure was investigated by employing different categories of simple and complex odor databases. Feature data sets were generated from the dynamic and steady state responses of the sensor array to the applied odor databases. Combinations of different feature extraction and classification methods were used to detect target gases. Validation of each technique was evaluated. Achievements of the study proved high classification rates of the fabricated e-nose in odor identification. It was indicated that gas identification is possible by applying the early selected portion of transient responses of the developed sensor array. The ability of the mentioned structure in analyzing gas mixtures was also investigated. The results presented high accuracy in the classification of gas mixtures.
\end{abstract}

Keyword: Conductive gas sensor; Electronic nose; Gas diffusion; Transient response 